\title{
Response Timing Detection Using Prosodic and Linguistic Information for Human-friendly Spoken Dialog Systems
}

\author{
Norihide Kitaoka \\ Masashi Takeuchi \\ Ryota Nishimura \\ Seiichi Nakagawa
}

Toyohashi University of Technology

(affiliation as previous author)

takeuchi@slp.ics.tut.ac.jp, http://www.slp.ics.tut.ac.jp

(affiliation as previous author)

nishimura@slp.ics.tut.ac.jp, http://www.slp.ics.tut.ac.jp

(affiliation as previous author)

nakagawa@slp.ics.tut.ac.jp, http://www.slp.ics.tut.ac.jp

keywords: prosodic information, surface linguistic information, response timing, human-friendly spoken dialog system

Summary

If a dialog system can respond to the user as reasonably as a human, the interaction will become smoother. Timing of the response such as back-channels and turn-taking plays an important role in such a smooth dialog as in human-human interaction. We developed a response timing generator for such a dialog system. This generator uses a decision tree to detect the timing based on the features coming from some prosodic and linguistic information. The timing generator decides the action of the system at every 100 ms during the user's pause. In this paper, we describe a robust spoken dialog system using the timing generator. Subjective evaluation proved that almost all of the subjects experienced a friendly feeling from the system.

\section{Introduction}

In Japanese human-human dialog, well-timed responses such as 'aizuchi' (sometimes called 'back-chan$n e l ')$ and turn-taking make for smooth dialog. Maynard [Maynard 93] said that aizuchi was the signal to make hearers continue to speak, to indicate his/her understanding of the utterances, and to express assent to them. Mizutani [Mizutani 01] said that aizuchi played the role of a "green light" to indicate permission for the hearer to continue his/her turn. As for turn-taking, one begins talking just after a pause of an appropriate length, or sometimes overlaps the hearer's utterance. In natural human-human dialogs, it is easy to see that the humans cooperatively talk to each other using various timing of aizuchi and turntaking. One times his aizuchi to the hearer's speaking rate, and also expresses his emotions by the timing of aizuchi and turn-taking.

The purpose of this study is to generate natural re- sponse timing of aizuchi and turn-taking. We are developing a human-friendly spoken dialog system which can generate natural response timing during a dialog.

Some realtime aizuchi generation systems have been developed so far. Ward [Ward 00] pointed out that a low pitch region of longer than $150 \mathrm{msec}$ in an utterance led to an aizuchi and built an aizuchi generator based on this heuristic rule. Okato et al. [Okato 96] built a system to make aizuchi using models of specific pitch patterns for user's utterances. Noguchi et al. [Noguchi 98] proposed a method to make aizuchi using prosodic information such as changes of fundamental frequency at the end of utterances and pauses. Sato et al. [Sato 02] investigated a method to detect natural turn-taking timing. They adopted a decision tree based on prosodic information, linguistic information, and the system semantic analysis results. Concerning turn-taking, there are many systems which allow users to barge in duaring an utterance of the system [Hirasawa 99, Kamm 97], but the system response timing 
of aizuchi and turn-taking is not considered.

Our goal is to develop a cooperative spoken dialog system. For this purpose, we are investigating not only how to select the action the system takes after the user's utterance, but also how to create an interval between the user's utterance and the system action (that is, the timing of the action).

In this paper, we first investigate the timing of aizuchi and turn-taking in human-human dialog. We found that some prosodic and surface linguistic information should affect the system behavior. Second, we adopted an algorithm named $\mathrm{C} 4.5$ which could automatically construct a decision tree to detect the response timing based on the features derived from the information, and then developed a timing generator based on the decision tree [Takeuchi 03]. Finally, we developed a human-friendly spoken dialog system using the timing generator. The system had a very simple dialog control unit which consisted of an ELIZA-like response generator and a slot filling-based semantic analyzer.

\section{Response timing generator}

\subsection{Corpus}

We used an annotated dialog corpus to analyze humanhuman dialog [JSAI 99]. The corpus has 29 dialogs consisting of speech (L and $\mathrm{R}$ channel) and the annotation. An example of an annotated dialog corpus is shown in Figure 1. This annotation contains the onset and the offset times of each utterance. $\mathrm{P}$ tag represents the pause and the number expressing the duration of the pause. In this paper, 11 dialogs of 3 tasks were used, and the total length of the dialogs was 47 minutes. These dialogs consisted of three tasks: chat, travel navigation and telephone shopping. Figure 2 shows the histograms of pause length between aizuchi and the preceding utterance, turn-taking and the preceding utterance, along with the utterances of the same speaker. We readily find that we cannot know the natural timing based only on the pause length from the preceding utterance.

We found that $63 \%, 35 \%$ and $18 \%$ of aizuchi in the dialogs of chat, travel navigation and telephone shopping, respectively, overlapped the preceding utterances. However, as described in this section, there are many cues at the end of the preceding utterances for timing generation, and we mainly adopt these cues to our system. Because of this situation, we do not deal with overlap in this paper, and the solution of

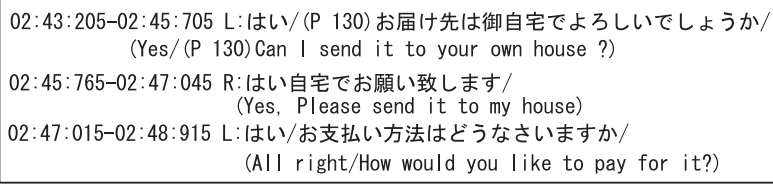

Fig. 1 An example of annotated dialog

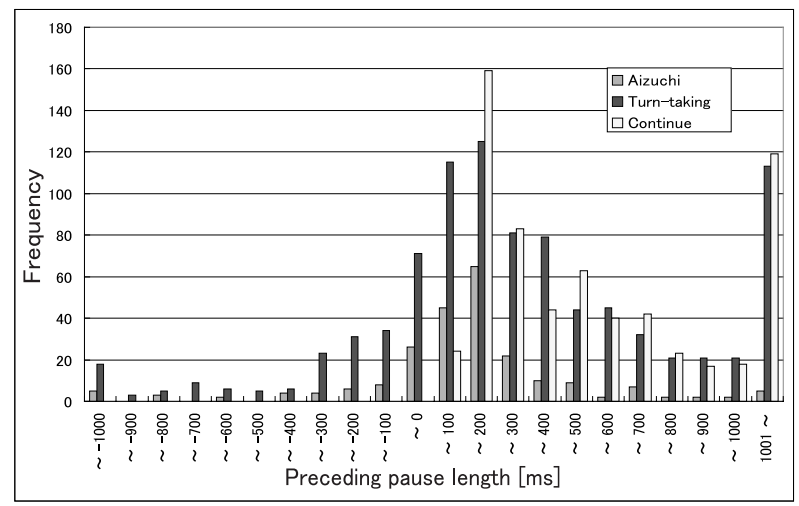

Fig. 2 Histogram of preceding pauses

this problem is a task for future investigation.

\subsection{Information in speech related to response timing}

\section{$\S 1$ Prosodic information}

Koiso et al. [Koiso 98] found prosodic cues to decide whether or not to make aizuchi. There are some particular pitch and power contour patterns of the last one mora of an utterance to make the opposite speaker (HEARER) generate an aizuchi.

On the other hand, Geluykens et al. [Geluykens 94] and Hirschberg [Hirschberg 02] showed in terms of turn-taking that fundamental frequency and the range are correlated with turn final versus turn keeping utterances in each phrase.

Okato et al. [Okato 96] mentioned that the duration of the utterance is also related to aizuchi. The longer the utterance is, the more frequently aizuchi occurs.

\section{$\S 2$ Linguistic information}

In Japanese dialog, turn-taking often occurs when the last word in the last utterance is a particle, an auxiliary verb, a verb or an interjection. We analyzed a corpus used in Section $2 \cdot 3$ and found that turn-taking was caused by the particles "ne" (chat task) and "ka" (other tasks) placed at the end of the utterance. This indicates that the last particles of utterances are related to trigger turn-taking. Topic-related phrases and some keywords also lead to aizuchi and turn-taking. For example, in telephone shopping, aizuchi often occurs just after a key word 


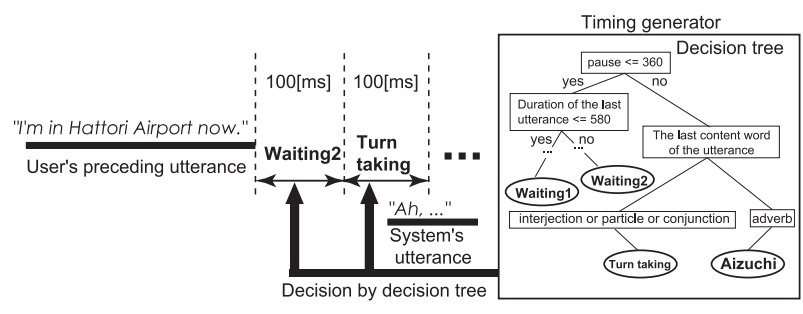

Fig. 3 Aizuchi and turn-taking generated by the decision tree

such as a product name.

\subsection{Response timing generator using prosodic and linguistic information}

In this section, we explain how to develop a response timing generator. As mentioned in Section $2 \cdot 1$, we do not deal with overlaps. The system decides the action and the timing of the action when detecting a user's pause. Actually, pauses of over a few dozen milliseconds in length can be easily detected under a high signal-to-noise ratio condition. In the experiments described in this section, however, we used manually labeled data instead of automatic detection.

Generally, the system automatically obtains surface linguistic information by speech recognition, and the speech recognizer can output the recognition results (or intermediate hypotheses) with small latency. ${ }^{* 1}$ Considering this situation, we assumed that the correct word sequences were obtained just at the end of the user's utterances. We wanted to know the performance of the generator with optimal information, so we used manual transcriptions instead of the recognition results in this section.

Almost all the cues for the aizuchi and turn-takings mentioned in Section $2 \cdot 2$ did not depend on the tasks. To apply the generator to various tasks, it is better not to depend on the tasks. Thus we did not divide the corpus by task.

\section{$\S 1$ Construction of response timing generator}

We developed a response timing generator using prosodic and linguistic information. The timing generator first detects a pause of the user, and then classifies every 100-ms segment of the pause into 4 classes: making aizuchi, taking the turn, waiting for user's successive utterance (turn-keeping; waiting (1)) and waiting for aizuchi or turn-taking (waiting (2)). This procedure is illustrated in Figure 3.

For the classification, we adopted the C4.5 learn-

*1 We explain how to output the hypotheses in realtime in Chapter 3. ing algorithm [Quinlan 92]. C4.5 can treat discrete and continuous values simultaneously. It constructs a decision tree, and we can know the decision process by looking at the tree. This algorithm can automatically construct a decision tree to classify the segments when given examples with the following features, and the tree can be converted to a set of decision rules:

(1) duration of preceding utterance

(2) part-of-speech of last word of preceding utterance

(3) kind of last postposition in preceding utterance

(4) elapsed time from end of previous utterance

(5) elapsed time from end of last content word

(6) duration of last content word in preceding utterance

(7) length between end of content word in preceding utterance and end of utterance

(8) fundamental frequency pattern of end of phrase

(9) power pattern of end of phrase

where features 2 and 3 were discrete, and the others were continuous. Features concerning durations (features 1, 4, 5, 6, and 7) were obtained from the manually labeled time information. Features based on surface linugistic information (features 2 and 3 ) were obtained from the manual transcriptions.

Patterns of fundamental frequency and power were described with first-order regression coefficients for fundamental frequency and power contours, respectively, in the last three regions of utterances obtained using 50-ms length sliding window with 25-ms overlap as shown in Figure 4. In each segment, we obtained fundamental frequencies by using the method in the study of Fujisaki [Fujisaki 90]. We used log-power (logarithm of RMS (root mean square)) as the value describing the power. These powers and fundamental frequencies were calculated every $6 \mathrm{~ms}$. These regression coefficients were calculated with a delay of 20-30 ms behind realtime, so the system could obtain them in time for the first decision point (50 ms, the middle point of the first segment in a pause). Decision by the decision tree took little time, so the system could process in realtime.

We prepared training data from a real spoken dialog corpus, which consisted of pause segments attached with tags of the correct answers (aizuchi, turntaking, waiting (1), waiting (2)) and the values of the features. To avoid over-fitting, we artificially added 


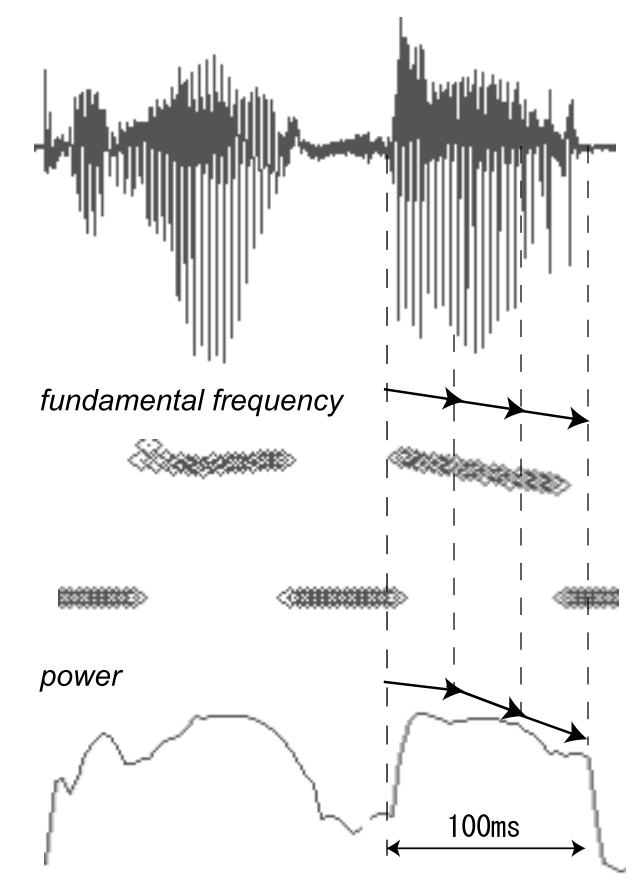

Fig. 4 Regression coefficients of fundamental frequency and power at the end of an utterance

aizuchi and turn-taking data with a real elapsed time $\pm 50 \mathrm{~ms}$ from the end of the previous utterance. The numbers of aizuchi and turn-taking were relatively fewer than those of waiting (1) and waiting (2), so we used aizuchi and turn-taking data repeatedly to standardize the occurrences of those data. Using this tree, the system can detect the action that the system should take at each segment.

We mentioned that topic-related phrases and keywords may be cues for the timing, but we do not deal with such information because we intend to make a task-independent system.

\section{$\S 2$ Analysis of decision tree}

Figure 5 shows a part of the decision tree made using the training set of all the tasks (chat, travel navigation, telephone shopping).

In this decision tree we found that most of the features appearing in the decision were prosodic, such as utterance duration, dynamics of fundamental frequency and power and pause durations after the preceding utterances.

Although the content word duration was a major feature, linguistic information did not play an important role among the features. The characteristics of this task-independent decision tree might be caused by the training data consisting of all the tasks. So we also constructed and examined a decision tree for each task.

These task-dependent decision trees were all similar

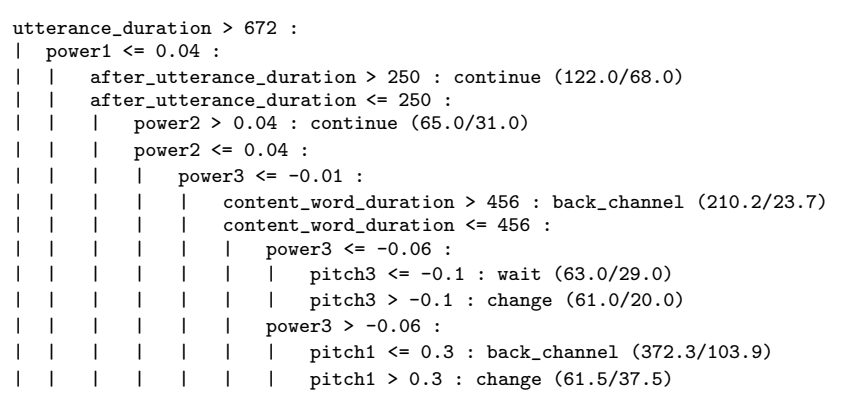

Fig. 5 A part of the decision tree trained by all the training data. (In this figure, power 1, 2, 3 and pitch 1, 2, 3 describe regression coefficients of log-powers and fundamental frequencies at the segments shown in Figure 4).

to the task-independent decision tree. This assured that the prosodic information played a more important role in detecting the response timing than linguistic information. Thus, we concluded that aizuchi and turn-taking can be detected only by prosodic information and a small amount of surface linguistic information (e.g., the distinction between content words and the others). One can also imagine that humans may be able to make aizuchi not depending much on the contents of preceding utterances.

\subsection{Evaluation of timing generator}

\section{$\S 1$ Timing reproduction experiment}

We evaluated the reproduction ability of the generator. In this experiment, we assumed that the correct word sequences were obtained just at the end of the user's utterances. In this section, we used all eleven dialogs for training and testing in the closed tests. As for the open test, we performed an 11-fold validation.

We compared the actions of actual humans and the system at each pause. For example, if the actual response was aizuchi at the third segment in a pause, and the system output was also aizuchi but at the fourth segment in the pause, then the classification was regarded as correct and the deviation was +1 segment. The classification accuracies are shown in Table 1 , and the histograms of deviations are described in Figure 6. In the table, recall rates, precisions, and F-measures are shown as well as the confusion matrix. The recall rate, precision, and F-measure mean the percentage of correctly discriminated pauses among the correct appearance, the percentage of correctly discriminated pauses among segments distinguished into target classes, and the harmonic average of the recall and the precision, respectively. The closed test result in Table 1 (a) shows that the response timing of the generator was similar to that of humans included 
Table 1 Pause classification accuracy (Top: confusion matrix; Bottom: recall, precision, and F-measure. Average of all the tasks)

(a) closed

\begin{tabular}{c|c|c|c}
\hline $\begin{array}{c}\text { System output } \\
\text { Actual responses }\end{array}$ & Aizuchi & $\begin{array}{l}\text { Turn- } \\
\text { taking }\end{array}$ & $\begin{array}{l}\text { Do } \\
\text { nothing }\end{array}$ \\
\hline Aizuchi & 152 & 13 & 7 \\
\hline Turn-taking & 47 & 573 & 77 \\
\hline Do nothing & 30 & 44 & 552 \\
\hline \hline Recall [\%] & 88.4 & 82.1 & 88.2 \\
\hline Precision [\%] & 66.1 & 91.0 & 86.8 \\
\hline F-measure & 75.6 & 86.3 & 87.5 \\
\hline
\end{tabular}

(b) open (11-fold)

\begin{tabular}{c|c|c|c}
\hline $\begin{array}{c}\text { System output } \\
\text { Actual responses }\end{array}$ & Aizuchi & $\begin{array}{c}\text { Turn- } \\
\text { taking }\end{array}$ & $\begin{array}{l}\text { Do } \\
\text { nothing }\end{array}$ \\
\hline Aizuchi & 50 & 41 & 81 \\
\hline Turn-taking & 81 & 319 & 298 \\
\hline Do nothing & 71 & 125 & 431 \\
\hline \hline Recall [\%] & 29.1 & 45.7 & 68.7 \\
\hline Precision [\%] & 24.8 & 65.8 & 53.2 \\
\hline F-measure & 26.7 & 53.9 & 60.0 \\
\hline
\end{tabular}

in the training data.

In contrast to the closed test, the open test (11fold validation) result of Table 1 (b) and Figure 6 (b) shows less consistency between the system and humans who did not belong to the training set than for the case of the closed test.

\section{$\S 2$ Discussion}

We made decision trees using 8 out of the 9 features described in Section $2 \cdot 3 \cdot 1$ and compared the degradations of reproduction performance from the original feature set. When not using features 1 (duration of the preceding utterance), 2 (part-of-speech of the last word of the preceding utterance), 4 (elapsed time from the end of the previous utterance), or 9 (power pattern of the end of the phrase), the performance was much degraded. Three of those four features were based on prosodic information. This supported the discussion in Section $2 \cdot 3 \cdot 2$.

The open test result was inferior to the closed test result in this reproduction experiment. It should be mentioned that these results did not imply the defectiveness of our method. Humans also have individual differences in this timing generation. Table 2 shows the agreement of aizuchi timing between the corpus and subjects. We replaced aizuchi in the corpus with pauses, and subjects pointed out the timing at which aizuchi should be made. Then we compared the positions of the subjects and the corpus. As shown in

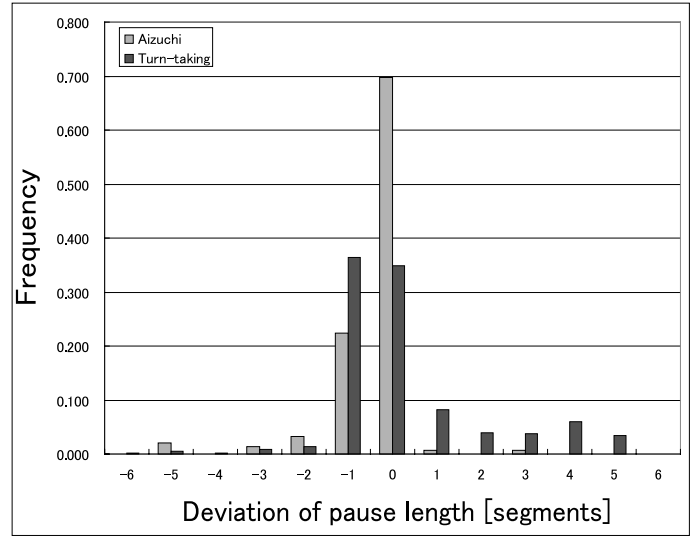

(a) closed

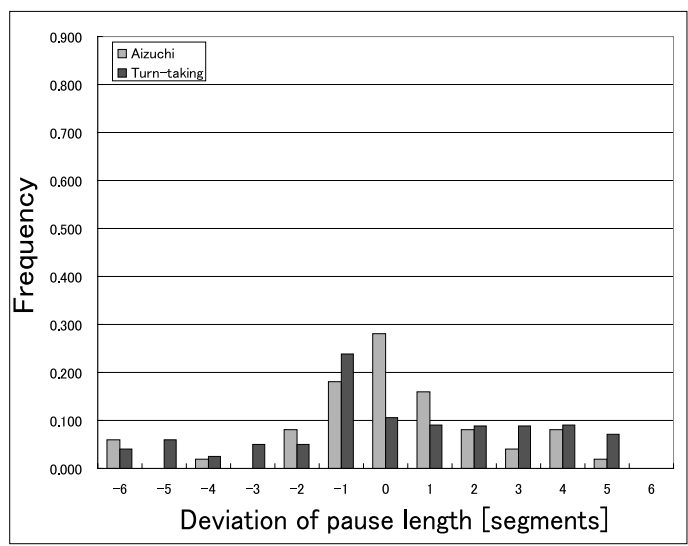

(b) open (11-fold)

Fig. 6 Histograms of deviations in segments between actual responses and system outputs correctly discriminated.

Table 2, a subject different from the person in the corpus could recall the aizuchi in the corpus at most at the rate of about $50 \%$ by hearing the speech of one side of the dialog in the corpus. These rates were comparable with the rates in Table 1 (b). This indicated that rates of agreement between human subjects were not so high, and that low agreement did not imply any lack of naturalness. Hence, we cannot evaluate the generator only by this objective test, and the timing by our generator may also be natural. We will confirm this fact in the next section.

\section{$\S 3$ Subjective evaluation}

To evaluate the naturalness of the timing by our generator, we performed a subjective evaluation.

We inserted an aizuchi extracted from the dialog of the same speaker who played the role of a system at the aizuchi timing point generated by our timing generator. We also made samples of turn-taking, but it was almost impossible to insert an alternative utterance which could appropriately respond to the subject's preceding utterance. Thus, we picked some filled pauses such as "Etto" (used like "aah ... let's 
Table 2 Agreement in aizuchi timing between subjects and corpus [\%]

\begin{tabular}{c|c|c|c}
\hline subject & task & recall & precision \\
\hline \multirow{3}{*}{ subject 1 } & chat & 50.0 & 47.6 \\
& travel navi & 36.8 & 35.0 \\
& telephone & 37.5 & 8.6 \\
\hline \multirow{3}{*}{ subject 2 } & chat & 20.0 & 57.1 \\
& travel navi & 5.3 & 14.3 \\
& telephone & 0.0 & 0.0 \\
\hline \multirow{3}{*}{ subject 3 } & chat & 5.0 & 4.8 \\
& travel navi & 61.4 & 33.3 \\
& telephone & 25.0 & 3.8 \\
\hline \multirow{3}{*}{ subject 4 } & chat & 20.0 & 50.0 \\
& travel navi & 29.8 & 38.6 \\
& telephone & 12.5 & 6.7 \\
\hline
\end{tabular}

see" in English), which is often employed at the beginning of an utterance, to insert at the time of system formation. Subjects listened to inserted aizuchi with a few preceding sentences and evaluated only the timing.

We compared the timing by the generator to that in the corpus. In real dialogs of the corpus, responses may have some meaning consistent with the context and the meaning may make subjects feel natural, especially in the case of turn-taking. To make subjects evaluate only the timing, we also replace the real response with aizuchi and a filled pause extracted from other parts of the dialog, as in the case of the generator.

We made 18 and 16 samples for the aizuchi timing of humans and the system, respectively, and 16 and 17 samples for turn-taking of humans and the system, respectively. Five persons listened to the data and made an evaluation by choosing one of the following: 1. too early timing; 2 . early; 3 . good; 4. late; 5. too late; and 6 . outlier (aizuchi or turn-taking should not occur at this pause). Results are shown in the first and second rows (Rep) of Table 3 and Table 4 for aizuchi and turn-taking, respectively.

We cannot find a significant difference between human (Corpus) and system, so in most cases our generator can generate natural timing if prosodic and surface linguistic information is available. We find that some samples felt too unnatural even in cases of human timing.

We also tested random and fixed timing of aizuchi and turn-taking. We picked 20 pauses for each test and inserted aizuchi and filled pauses. For random timing tests, the distribution of the timing was as in
Table 3 Subjective evaluation result (aizuchi). "rate" is the rate of natural timing. "Rep" means aizuchi replaced with the other one.

\begin{tabular}{c|c|c|c|c|c|c||c}
\hline & $\begin{array}{c}1 \\
\text { early }\end{array}$ & 2 & $\begin{array}{c}3 \\
\text { good }\end{array}$ & 4 & $\begin{array}{c}5 \\
\text { late }\end{array}$ & $\begin{array}{c}6 \\
\text { outlier }\end{array}$ & $\begin{array}{c}\text { rate } \\
{[\%]}\end{array}$ \\
\hline Corpus (Rep) & 0 & 12 & 66 & 9 & 0 & 3 & 73.3 \\
System (Rep) & 0 & 15 & 64 & 0 & 0 & 1 & 80.0 \\
Random & 5 & 15 & 56 & 15 & 7 & 2 & 56.0 \\
Fixed & 5 & 35 & 56 & 1 & 1 & 2 & 56.0 \\
Corpus (Real) & 1 & 13 & 69 & 6 & 0 & 6 & 72.6 \\
\hline
\end{tabular}

Table 4 Subjective evaluation result (turn-taking). "Rep" means turn-taking replaced with a filled pause.

\begin{tabular}{c|c|c|c|c|c|c||c}
\hline & $\begin{array}{c}1 \\
\text { early }\end{array}$ & 2 & $\begin{array}{c}3 \\
\text { good }\end{array}$ & 4 & $\begin{array}{c}5 \\
\text { late }\end{array}$ & $\begin{array}{c}6 \\
\text { outlier }\end{array}$ & $\begin{array}{c}\text { rate } \\
{[\%]}\end{array}$ \\
\hline Corpus (Rep) & 0 & 15 & 54 & 7 & 0 & 4 & 67.5 \\
System (Rep) & 3 & 21 & 55 & 1 & 0 & 5 & 64.7 \\
Random & 2 & 25 & 44 & 18 & 7 & 4 & 44.0 \\
Fixed & 5 & 21 & 66 & 4 & 1 & 3 & 66.0 \\
Corpus (Real) & 0 & 6 & 72 & 2 & 0 & 0 & 90.0 \\
\hline
\end{tabular}

Figure 2, but overlaps were excluded. For fixed timing tests, the timing was the averages of the Figure 2 (108 ms for aizuchi and $268 \mathrm{~ms}$ for turn-taking). The results are shown in the third and fourth row of Table 3 and Table 4 . We found significant degradations from the system cases except for the fixed timed turntaking.

Then, we tested the real aizuchi and turn-taking to examine the effect of the replacement. For this experiment we used 19 and 16 samples for aizuchi and turn-taking, respectively.

The results are shown in the fifth rows (Real) of Table 3 and Table 4 for aizuchi and turn-taking, respectively. Even if subjects heard a real response, the aizuchi was not always evaluated as natural. This suggests the difficulty of aizuchi timing evaluation by third parties. Individual differences may cause such unnaturalness. A certain person's aizuchi may make someone else feel unnatural. Naturalness of the turntaking may rely heavily on the contents of the succeeding utterance. The result of turn-taking in Table 4 supports the hypothesis.

\section{Dialog system with response timing generator}

\subsection{System architecture}

Almost all the spoken dialog systems could not react to the user's utterance in realtime, and the user could not know whether or not the system really listens to the user's utterance. Nowadays, the systems can immediately respond to the users because of the speeding up of computers, but the appropriate timing is not under consideration. Recently, Fujie et al [Fujie 04] proposed an architecture which generates aizuchi and repetition of the user's word using finite-state transducer-based speech recognizer and prosodic information-based timing extractor. However, their sys- 


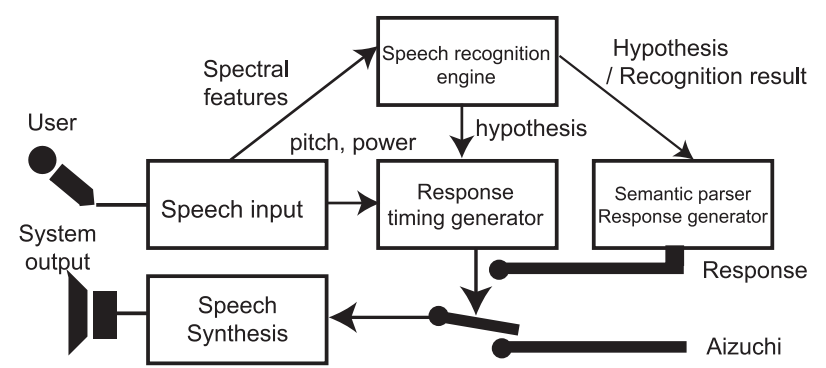

Fig. 7 Our target system

tem does not consider turn-taking.

We propose a new spoken dialog system architecture adopting a response timing generator. The proposed system architecture is shown in Figure 7 . The speech input module extracts spectral features, pitch and power parameters. Spectral feature parameters are sent to a speech recognition engine, while pitch/ power parameters are sent to a response timing generator. The CFG-driven speech recognition engine SPOJUS [Kai 92] outputs intermediate hypotheses in realtime. When intermediate hypotheses are needed (that is, a pause appears), SPOJUS checks the likelihood of the best hypothesis which is ended with a word at the time; if the likelihood is sufficiently high, then SPOJUS outputs the hypothesis with the word alignment information. When the user utterance finishes, SPOJUS also outputs the final recognition results with word alignment. The response timing generator selects the action of the system from among waiting, making aizuchi and taking the turn using the hypotheses from the speech recognition engine and pitch/power parameters from a speech input module at every $100 \mathrm{~ms}$ segment in the user's pause. The semantic parser generates a semantic representation progressively using a simple slot-filling method activated by keywords in the recognition results, and prepares a response sentence based on this semantic representation. This parser also prepares another response sentence using an ELIZA-like method [Weizenbaum 65]. The response generator can select an appropriate response from these sentences. This strategy can continue the dialog even if the semantic representation is not constructed enough to generate a response sentence. The system has some exceptional rules to continue the dialog: for example, the system prompts the user to speak something after a long pause (6 seconds in our system).

\subsection{Example of dialog system}

One of our system's goals is to become very familiar so that humans will chat with it. We chose the weather information service as the task (http:/ /www.imoc.co.jp/yohou.htm), because weather is a popular conversational topic and the vocabulary range is not so large. We used a vocabulary of 300 words.

An example of a dialog with the system is shown in Figure 8. The system sometimes made aizuchi and also uttered "rambling" sentences (for example, "Is it hot in Toyohashi?").

Four male subjects talked with the system about the weather. We did not give any instruction about the constraints for speaking styles or vocabulary, etc. The only direction was to obtain certain weather information desired from the system. The dialog was aborted if the subject could not obtain the desired information even after a long talk with the system.

Four types of system were compared. About the timing generation, we compared the system with that of humans. In the latter case, the human operator only generated the timing, while the contents of responses were generated by the system. We also compared the systems with/without aizuchi. We used only "hai" as aizuchi. We informed the subjects in advance as to whether or not the system could make aizuchi, but did not inform them as to whether the timing was generated by the system or a human operator.

Subjects answered a questionnaire right after each dialog. We encouraged them not to mind the contents of the system's utterance when evaluating the timing of responses. This may be summarized as follows.

The timing and frequency of system's aizuchi were fairly good. This showed that the timing detection of aizuchi was robust against recognition errors, etc. But the familiarity of the system with aizuchi was inferior to that without aizuchi. An aizuchi with inadequate timing tended to make subjects feel more uncomfortable than turn-taking with inadequate timing. The timing generation performance depended on the realtime pitch/power detection and the speech recognition with errors, causing inadequate timing. The improvement of performance is a task for further investigation.

All the subjects preferred the system with aizuchi when the response timing was generated by a human operator, encouraging us to improve this system. 


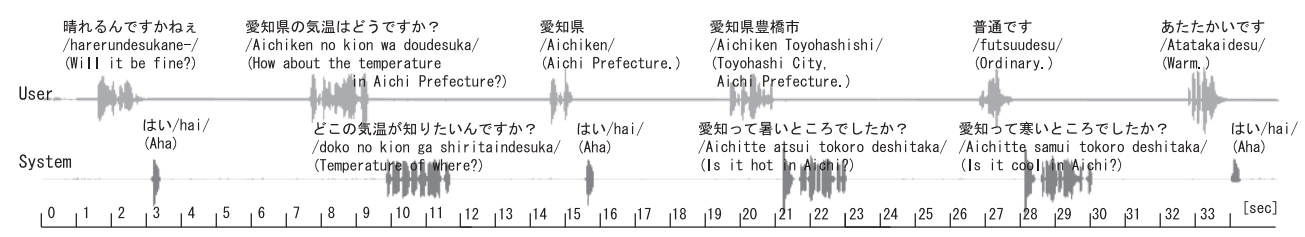

Fig. 8 An example of a dialog between the system and a user.

\section{Conclusions}

We developed a dialog system which can generate timing of aizuchi and turn-taking in realtime. The system had the response timing generator using a decision tree based on prosodic and surface linguistic information.

We obtained a good rating for the timing generator itself, but not for timing in a spoken dialog system because of the limitation of realtime processing of prosodic and linguistic features. Nevertheless, the dialog system was preferred because of its frequent and quick response, which enabled users to know that the system really listens to their utterances.

Subjects pointed out that a response other than 'hai' should be used as aizuchi. They also indicated that the system sometimes repeated the same content and the dialog could not proceed. We must therefore improve not only the timing generator but also the sentence generator for a greater variety of sentences.

In the future, our timing generator must be improved investigating machine learning techniques other than decision tree. As for features, Osuga et al. [Osuga 04] suggests that the features related to the fundamental frequency, the power and the duration of not only the last mora but the other parts of the preceding utterance, are informative for timing detection. We adopt more features including these for our generator, which cannot make a response which overlaps the preceding user utterance. We thus plan to extend the generator and the dialog system to support the overlaps.

\section{Acknowledgments}

This research has been supported in part by Scientific Researchs on Priority Areas: Prosody and Speech Processing (No. 12132205), and Informatics (No. 16016244).

\section{$\diamond$ References $\diamond$}

[Maynard 93] S. K. Maynard, Kaiwa Bunseki, Kuroshio Shuppan, (in Japanese) 1993.

[Mizutani 01] N. Mizutani, "Aizuchi to Pause no shinrigaku", Gengo, pp. 46-51 (in Japanese), Jun, 2001.
[Ward 00] N. Ward, "Prosodic features which cue backchannel responses in English and Japanese", Journal of Pragmatics, vol. 32, pp. 1177-1207, 2000.

[Okato 96] Y. Okato, K. Kato, M. Yamamoto, and S. Itahashi, "Insertion of interjectory response based on prosodic information" IEEE Workshop Interactive Voice Technology for Telecommunication Applications (IVTTA-96), pp. 85$88,1996$.

[Noguchi 98] H. Noguchi, Y. Den, "Prosody-based detection of the context of backchannel responses", ICSLP-98, pp. 487-490, 1998.

[Sato 02] R. Sato, R. Higashinaka, M. Tamoto, M. Nakano, K. Aikawa, "Learning decision trees to determine turntaking by spoken dialogue systems", ICSLP-02, pp. 861864, 2002.

[Takeuchi 03] M. Takeuchi, N. Kitaoka, S. Nakagawa, "Generation of natural response timing using decision tree based on prosodic and linguistic information", EUROSPEECH2003, pp. 609-612, 2003.

[Takeuchi 04] M. Takeuchi, N. Kitaoka, S. Nakagawa, "Timing detection for realtime dialog systems using prosodic and linguistic information", International Conference: Speech Prosody 2004, pp. 529-532, 2004.

[Hirasawa 99] J. Hirasawa, M. Nakano, T. Kawabata, and K. Aikawa, "Effects of system barge-in responses on user impressions", EUROSPEECH-99, Vol 3, 1391-1394, 1999.

[Kamm 97] C. Kamm, S. Narayanan, D. Dutton, and R. Ritenour, "Evaluating spoken dialogue systems for telecommunication services", EUROSPEECH-97, pp. 2203-2206, 1997.

[Geluykens 94] R. Geluykens, M. Swerts, "Prosodic cues to discourse boundaries in experimental dialogues", Speech Communication, vol. 15, pp. 69-77, 1994.

[Hirschberg 02] J. Hirschberg, "Communication and prosody: functional aspects of prosody", Speech Communication, vol. 36, pp. 31-43, 2002.

[Kai 92] A. Kai and S. Nakagawa, "A frame-synchronous continuous speech recognition algorithm using a top-down parsing of context-free grammar", ICSLP-92, pp. 257-260, 1992.

[Weizenbaum 65] J. Weizenbaum, "ELIZA - A computer program for the study of natural language communication between man and machine", Communications of the ACM, vol. 9, no. 1, pp.36-45, 1965.

[JSAI 99] SIG of Corpus-Based Research for Discourse and Dialogue, JSAI, "Constructing a spoken dialogue corpus as sharable research resource", Japanese Society for Artificial Intelligence, SIG-SLUD-9903-4, 1999.

[Koiso 98] H. Koiso, Y. Horiuchi, S. Tutiya, A. Ichikawa, and Y. Den, "An analysis of turn-taking and backchannels based on prosodic and syntactic features in Japanese map task dialogs", Language and Speech, vol.41, No.3-4, pp. 291-317, 1998.

[Quinlan 92] R. J. Quinlan, "C4.5:Programs for machine learning", Morgan Kaufmann, 1992.

[Fujisaki 90] H. Fujisakki, K. Hirose, S. Seto, "A scheme for pitch extraction of speech using autocorrelation function with frame length proportional to time lag," IEICE Technical Report, SP90-86, pp.9-16, 1990.

[Fujie 04] S. Fujie, K. Fukushima, D. Shibata, T. Kobayashi, "Conversation robot with feedback/repetition function based on FST and prosody information," JSAI SIG-SLUD- 
A401-03, 2004

[Osuga 04] T. Ohsuga, M. Nishida, Y. Horiuchi, A. Ichikawa,

"Role of prosody in turn-taking in Japanese spontaneous dialogue," JSAI SIG-SLUD-A401-07, 2004.

〔担当委員 : 奥乃 博〕

Received November 3, 2004.

\section{Author's Profile}

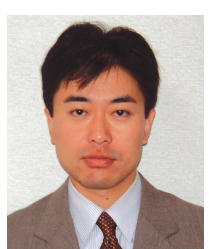

Kitaoka, Norihide (Member)

received his B.E. and M.E. degrees from Kyoto University in 1992 and 1994, respectively, and a Dr. Eng. degree from Toyohashi University of Technology in 2000. He joined DENSO CORPORATION, Japan in 1994. He then joined the Department of Information and Computer Sciences at Toyohashi University of Technology as a research associate in 2001 and has been a lecturer since 2003. His research interests include speech processing, speech recognition and spoken dialog. He is a member of the Institute of Eleectronics, Information and Communication Engineers (IEICE), the Information Processing Society of Japan (IPSJ), and the Acoustical Society of Japan (ASJ).

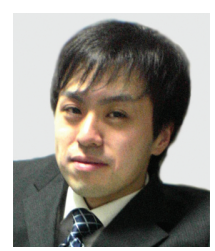

Takeuchi, Masashi

received his B.E. and M.E. degrees from Toyohashi University of Technology in 2002 and 2004 , respectively. His research interests include spoken dialog systems. He joined the Sony Corporation in 2004 .

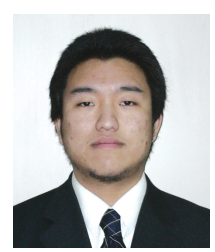

\section{Nishimura, Ryota}

received his B.E. degree from Toyohashi University of Technology in 2005 and is currently a graduate student of Toyohashi University of Technology. His research interests include spoken dialog systems.

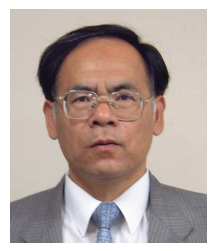

\section{Nakagawa, Seiichi (Member)}

received his B.E. and M.E. degrees from Kyoto Institute of Technology, in 1971 and 1973, respectively, and a Dr. of Eng. degree from $\mathrm{Ky}$ oto University in 1977 . He joined the faculty of Kyoto University, in 1976, as a Research Associate in the Department of Information Sciences. From 1980 to 1983 he was an Assistant Professor, and from 1983 to 1990 an Associate Professor. Since 1990 he has been a Professor in the Department of Information and Computer Sciences, Toyohashi University of Technology, Toyohashi. From 1985 to 1986, he was a Visiting Scientist in the Department of Computer Science, Carnegie-Mellon University, Pittsburgh, USA. He received the 1997/2001 Paper Award from the IEICE and the 1988 JC Bose Memorial Award from the Institution of Electro. Telecomm. Engrs. His major interests in research include automatic speech recognition/speech processing, natural language processing, human interface, and artificial intelligence. 